\title{
Quand Érasme se fait politique: la première lettre à Sigismond $1^{\mathrm{er}}$ le Vieux
}

\section{DANIÈLE LETOCHA}

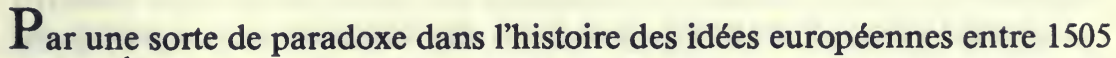
et $1565,{ }^{1}$ la figure d'Érasme paraît être à la fois omniprésente et absente. Omniprésente en ce qu'on imagine mal un ouvrage traitant d'un aspect quelconque de l'humanisme ou de la Réforme qui ne fasse une large place à ses oeuvres et à sa correspondance. On fait alors état de "l'influence" globale d'Érasme sur les milieux lettrés en invoquant tous ceux qui se réclament de lui, sans distinguer les énoncés objectifs des projections fictives ou affectives dans la cacophonie des filiations putatives. Et c'est en tentant de réduire ces rapports à des parentés intellectuelles textuelles qu'on constate l'absence d'Érasme.

En effet, il est quasi impossible d'attribuer en propre au maître l'initiation d'une seule thèse, d'une seule conclusion thématique forte et tranchante; quasi impossible, également, de nommer un seul disciple qui eût reçu et développé une théorie érasmienne identifiable, c'est-à-dire discursivement opposable à d'autres. Comme le remarque Huizinga, ${ }^{2}$ l'auteur des Adagia ne nous a pas laissé une phrase assez dense pour qu'elle serve couramment de proverbe; on pourrait ajouter qu'il n'existe pas de citation d'Érasme immédiatement familière à un esprit formé à la "culture générale," ni au XVI ${ }^{e}$ siècle, ni aujourd'hui.

Dans ces conditions, qu'appelle-t-on "érasmianisme"? Quel est le référent exact de cette notion dans le corpus érasmien d'abord, puis chez d'autres penseurs? Nous faisons l'hypothèse qu'à la différence d'une théorie structurée, comme le machiavélisme en fournit un exemple, l'érasmianisme constitue un phénomène culturel fluide, d'ordre essentiellement herméneutique. Au XVIe siècle, on s'est proclamé disciple d'Érasme à partir 


\section{2 / Renaissance and Reformation}

d'identifications subjectives, en prélevant un ou plusieurs motifs dans la tapisserie érasmienne qui en compte une constellation indéfinie. Ce que nous appelons un motif érasmien, c'est un agrégat d'idées et de valeurs n'ayant pas le statut d'un concept, ni celui d'un constat empirique. Ces motifs ne forment pas un arrangement systématique cumulatif: ils demeurent ponctuels, cellulaires, et se déploient à l'horizontale sous le mode de la récurrence. C'est le cas, par exemple, de l'intention pédagogique ou de l'irénisme religieux. Pour s'approprier un motif, l'emprunteur doit le sortir de son contexte d'origine et l'insérer dans un autre par un jeu d'interprétations.

L'objectif ultime de ce projet, dans toute son extension en trois parties, est de fournir une lecture culturelle des perspectives souvent conflictuelles construites par les érasmiens polonais. Le résultat visé consiste en une topographie générale qui se fonde sur la répartition des motifs empruntés à Érasme et transformés par la suite. Le premier volet qui suit se limite à exposer les données événementielles concernant les rapports entre Érasme et la Pologne.

La déclaration d'Érasme "Polonia mea est," dans sa lettre à William Warham du 4 septembre $1524^{3}$ si souvent citée, témoigne que le prince des lettres se montrait sensible à l'accueil favorable fait à ses oeuvres dans les cercles humanistes de Cracovie ainsi que chez les magnats mécènes de province. Ce n'était pas là pure vanité: les faits donnent une indéniable substance à cette perception.

A compter des éditions cracoviennes de la Querela pacis et des Colloquia, sorties des presses de Haller en 1518, l'admiration et même l'engouement pour le discours érasmien domine dans les réactions des intellectuels polonais face aux apports étrangers, fort nombreux pendant cette période de crise religieuse. La fidélité polonaise ne se dément pas car une édition locale de l'Hyperaspistes rencontre plus tard un large succès et, jusqu'à l'installation des Jésuites en Warmie, en 1565, la sympathie pour Érasme prévaut aussi bien chez une majorité des professeurs de l'université Jagellonne que chez les aînés qui n'ont pas reçu de formation humaniste, ni séjourné dans les universités étrangères. On peut fixer en 1519 l'entrée de "l'esprit érasmien" à l'université Jagellonne en prenant pour point de repère les premiers cours de Leonard Cox sur les lettres de Jérôme; cet Anglais, ami d'Érasme et, comme la plupart des savants immigrés ou réfugiés en Pologne, fortement identifié à la polonité, utilisait le texte jérômien établi par Érasme ${ }^{4}$ et enseignait les méthodes philologiques de ce dernier avec le 
plus grand succès. Une telle convergence des appuis institutionnels constitue une exception dans l'Europe du temps. Cela peut s'expliquer par la genèse de la culture polonaise, particulièrement par les contraintes socio-politiques et ethno-religieuses qui avaient façonné des perspectives d'ouverture originales dont nous avons rendu compte ailleurs. ${ }^{5}$ Retenons que, jusqu'à la mort d'Érasme, au moins, l'Université, la cour et le haut-clergé manifestent une rare unanimité pour célébrer ses mérites littéraires et moraux, ce qui ne s'observait ni aux Pays-Bas, ni en France, ni dans l'Empire et encore moins en Espagne. D'ailleurs, en Pologne, l'intelligentsia de l'époque est quantitativement trop limitée pour être fortement segmentée et ces trois "milieux" auxquels on peut ajouter celui de la banque se rencontrent autour de l'académie humaniste présidée par Andrzej Trzecieski l'aîné, admirateur d'Érasme. ${ }^{6}$

Entre 1523 et 1536, la correspondance connue entre Érasme et l'élite polonaise compte quatre-vingt-douze échanges dans les deux sens. Avec Andrzej Krzycki (Cricius), Piotr Tomicki (Tomicius), Jost Decjusz (Dietz, dit Decius), et Jan Antonin, ce sont les membres de la famille Laski qui s'avèrent les plus présents parmi les correspondants d'Érasme. Quelques précisions sur ce clan de petite noblesse que les rois Jagellons ont élevé à une situation de puissance intellectuelle, ecclésiastique, politique et diplomatique: il s'agit d'abord de l'évêque Jan Laski, devenu primat de l'Église polonaise en 1510, auquel Érasme dédia son édition d'Ambroise, et qui n'a pas lui-même reçu d'initiation aux lettres classiques mais a assuré une formation humaniste de qualité à ses trois neveux: Hieronim, diplomate qui avait passé sa jeunesse au service de la France et partagé la captivité de François $1^{\text {er }}$ en Espagne, Jan le Réformateur (Johannes a Lasco) ${ }^{7}$ le plus connu en Occident, et Stanislaw, ${ }^{8}$ expert en politique orientale et négociateur auprès des Turcs. Seweryn et Jan Boner, issus de la banque, mécènes et alliés à la famille Laski, sont également des correspondants d'Érasme. Decjusz, originaire de Basse-Alsace, fournit un excellent exemple de circulation d'un milieu à l'autre: d'abord venu en Pologne, au service de la maison Boner qui détenait du roi des droits d'exploitation minière, Decjusz devint, quatorze ans plus tard, secrétaire du roi dont il se fit l'historiographe dans le $D e$ Sigismundi temporibus, et diplomate d'importance. Son cas illustre la règle selon laquelle l'érudition devient un facteur d'ascension sociale et, pour la Pologne, de promotion économique.

Une série de contacts personnels initia ces liens épistolaires. Le primat envoya d'abord, en 1520, son neveu Hieronim Laski en ambassade auprès 
de Charles Quint pour lui exprimer les voeux de Sigismond I le Vieux, à la suite de l'élection impériale de l'année précédente, quoique le roi et le primat fussent tous deux des adversaires acharnés des Habsbourg et qu'ils eussent soutenu la candidature de François $1^{\text {er }}$. Hieronim avait alors rẹu la mission de rencontrer Érasme qu'il vit une première fois à Bruxelles, puis à Cologne.9 Il le revit en 1524, à Bâle, et se joignit à ceux qui pressaient Érasme de se prononcer contre Luther. De nouveau, l'année suivante, Hieronim lui rendit visite et lui présenta son frère Jan Laski le Réformateur qui établit aussitôt des rapports personnels et politiques suivis avec le maitre, chez lequel il demeura pour un séjour.

Les relations entre Érasme et le jeune Jan Laski furent marquées par deux événements majeurs, l'un de portée internationale, l'autre d'ordre privé. Arrêtons-nous plus longuement sur le premier que j'appelle "la lettre du malentendu."

Le contexte est celui du règlement politique par le Roi Sigismond I le Vieux (1506-1548) de la question prussienne. Installés sur la côte du nord polonais depuis $1226^{10}$, grâce à une entente ${ }^{11}$ constamment recusée $e^{12}$ par la couronne, les Chevaliers Teutoniques avaient été vaincus à Grünwald, en 1410 , par le premier roi Jagellon. Mais cette victoire militaire n'avaient été - ni territorialement achevée, ni politiquement exploitée. C'est ainsi que les conflits séculaires avaient repris ${ }^{13}$ et que Sigismond s'était remis en campagne. Lorsqu'il accepta une trève de quatre ans, en 1521, c'était avec l'intention d'en finir avec l'encerclement de l'Empire qui progressait depuis la Bohême jusqu'à la frontière de la Samogitie, épuisant des troupes nécessaires pour contenir les Moscovites. C'est ce moment que choisit le grand maître Albert de Brandebourg (Hohenzollern de la branche cadette) pour séculariser son ordre et se déclarer duc héréditaire de Prusse, dès lors chef d'un état passé en bloc au luthéranisme, appliquant à l'avance la maxime d'Augsbourg: "Cujus regio, hujus religio." Du fait même, il perdit l'appui de Charles Quint qui le mit au ban de l'Empire. ${ }^{14}$ Voyant la faiblesse de cette position prussienne, Sigismond hésita devant une guerre finale qui eât pu liquider la question. ${ }^{15}$ Il arrêta enfin son choix sur une solution négociée à deux volets. Une partie de la Prusse fut intégrée à la couronne polonaise et prit le nom de Prusse royale; ${ }^{16}$ Albert gardait autorité sur le reste du territoire, la Prusse ducale, qu'il déclara vassale du roi de Pologne par le traité de Cracovie. C'est ainsi que le 10 avril 1525 , sur la place du marché de Cracovie, le duc Albert, un genou en terre, présenta l'hommage de la Prusse à Sigismond qui en fit un spectacle vengeur. 
Hieronim Laski avait pris une part active à l'élaboration de ce délicat traité. C'était là, en effet, un audacieux précédent: le premier traité de suzeraineté entre un État catholique et un État luthérien. Charles Quint tenta de l'empêcher, y voyant une trahison en plus d'une hérésie: la mise au ban dépouillait un sujet de tout pouvoir civil, dont celui de traiter et, d'autre part, l'association avec l'hérésie constituait un vice susceptible d'annuler l'acte. Johann Eck, l'adversaire de Luther, avait menacé Sigismond de représailles pour êmpecher la conclusion du traité. ${ }^{17} \mathrm{~A}$ la demande de la cour polonaise, Krzycki rédigea une justification, sous le titre de De negotio prutenico, qui alléguait que la sécularisation prusienne était un fait accompli ne laissant qu'une dimension de politique neutre au traité. L'argumentation de Krzycki postule l'autonomie de la raison d'État.

C'est dans ce climat qu'à l'automne de 1525, Jan Laski le Réformateur écrivit plusieurs fois ${ }^{18}$ à Érasme en le priant d'intervenir à l'appui de Sigismond et qu'il l'instruisit à Bâle des complexités du cas. Érasme, si étranger à tout engagement partisan et singulièrement allergique aux rivalités religieuses comme politiques, accepta. Lui qui était pensionné par Charles Quint à titre de conseiller et qui avait alors grand besoin d'une image acceptable à la curie de Rome, comprit-il vraiment la portée de son intervention? On peut en douter. Le 15 mai 1527, il adressa à Sigismond I le Vieux la célèbre lettre ${ }^{19}$ ou la piété, la prudence, la tolérance et le pacifisme du roi sont vantés comme de grandes vertus évangéliques autant qu'antiques.

A l'examen rigoureux du contexte et du texte de cette lettre, il devient évident qu'Érasme, alors dans ses années de maturité, n'a rien du penseur politique: non pas que le bon exercice du pouvoir le laisse indifférent, mais plutôt du fait qu'il lui manque concepts, modèles, analyses et théories des conflits entre nations, de leur causes et de leurs possibles résolutions. Ce qui lui fait d'abord défaut, c'est une claire représentation de sa propre situation objective et du type spécifique d'autorité que peut conférer la légitimation apportée par un intellectuel prestigieux à un délicat précédent politique. L'affaire paraît en effet scandaleuse, considérée sur l'horizon de ce premier tiers de siècle. Elle ouvre la porte aux intentions politiques nationales, coupées de tout égard pour le pouvoir spirituel et matériel de la papauté, tel ce traité commercial de 1528 entre François $1^{\text {er }}$ et les Turcs, lesquels assiègent Vienne l'année suivante.

En ce même mois de mai 1527 où Érasme prend la plume pour écrire à Sigismond, Charles Quint, mécontent de la politique pro-française de Clément VII, autorise le sac de Rome par les Impériaux. Prisonnier au 


\section{6 / Renaissance and Reformation}

château Saint-Ange, le pape ne sera libéré que contre forte rançon et le cardinal Wolsey, venu à Rome pour négocier le divorce d'Henry VIII sans avoir réussi à voir ce prisonnier, rentrera en Angleterre résigné au schisme. Dans ce monde bouleversé, l'espoir d'ordre paraît anéanti.

Dans sa lettre à Sigismond, Érasme, Batave d'Empire, épouse les intérêts polonais avec une extrême naiveté, fasciné qu'il est par le paradis de paix religieuse et civile - fort réelle - que constitue la Sarmatie. Il ne montre aucune distance, aucun recul sur la situation, aucune notion de ce qui rend la solution polonaise singulière et non transposable, enfin, aucune perception des conséquences de ses jugements sur les intérêts de son propre souverain. C'est un homme de livres et non un homme d'action, un moraliste et non un politique.

Voici donc un cas illustrant le caractère flou et même confus de l'érasmianisme surpris en dehors du champ philologique du prince des lettres entre 1524 et 1527 . Jusqu'à sa condamnation, on continuera de répéter que c'est lui qui a pondu l'oeuf que Luther a couvé. Le succès européen de L'Éloge de la folie l'a associé contre son gré à ceux que les critiques des institutions et pratiques romaines ont conduits à la rupture. Et ces derniers ne comprennent pas qu'Érasme ne rejoigne pas leur camp. On aurait tort, en effet, de sous-estimer la dureté des attaques d'Érasme contre le clergé: il en fustige l'ambition la plus crue, l'avarice insatiable, l'inextinguible soif de voluptés et la fureur guerrière. ${ }^{20}$ Coincé entre les dénonciations des catholiques et la violence des luthériens (non encore constitués en Église), il est aux abois et développe un sentiment, sinon un délire, de persécution. Il écrit a Warham, le 4 septembre 1524:

En réalité, j’ai affaire à trois armées. A Rome, certaines gens qui sont férus de littérature paienne me haissent furieusement....Certains théologiens et moines mettent tout en oeuvre pour me perdre. Mais personne ne gronde avec plus d'extravagance que ceux qui s'appellent luthériens. ${ }^{21}$

Deux ans auparavant, Érasme a dú quitter Louvain où ses livres dérangeaient. Il devra de même quitter Bâle, en 1529, en conflit avec Oecolampade dont il a, en 1525, jugé un traité "non conforme à la pensée de l'Église."22 Il lui faut évaluer les possibles refuges et peut-être Cracovie lui parut-elle alors un des hâvres à ménager. Cela n'est qu'une hypothèse. 
Quelques jours après avoir écrit à Sigismond, Érasme confie ses craintes à Jan Laski estimant que: "la vaste conjuration des pharisiens" veut sa peau. ${ }^{23} \mathrm{La}$ contre-attaque qu'il dit souhaiter, c'est que ses amis s'emploient à faire circuler ses oeuvres morales. Car il a fait de la simplicité évangélique sa réponse personnelle et universelle aux défis de son temps. A ses yeux, tout le mal vient des théologiens: une réponse théologique envenimerait donc des débats décadents, dangereux et superflus. C'est pourquoi il refuse de cristalliser la doctrine chrétienne en une orthodoxie servant d'arme contre l'hérésie qu'elle contribue à durcir. Reprenant un argument constamment invoqué par les tenants de la tolérance dogmatique, depuis l'époque des Pères, Érasme en appelle à la parabole de l'ivraie et du bon grain. ${ }^{24} \mathrm{Cet}$ anti-intellectualisme détermine la place d'Érasme non seulement en théologie, mais encore en philosophie et en politique. ${ }^{25}$ Le fait qu'il ne croie pas possible de fixer un critère de vérité intrinsèque en ces matières est décisif dans son type particulier d'irénisme et de tolérance. C'est cette épistémologie minimaliste qui lui fait dire: "d'ailleurs, plus il y a de dogmes, plus il y a matière à hérésie." ${ }^{26}$ Pour Érasme, ce sont la piété et la charité qui sauvent. Il faut comprendre que cette position ne fait rien en faveur de la clarification de son statut confessionnel, disputé par ses ennemis. Elle marque aussi la limite théorique des thèses d'Érasme et les contradictions où il doit s'engager. Pressé par les plus hautes instances, ce contempteur des querelles dogmatiques doit s'exécuter et adresser à Luther, en 1524, la rectification théologique qu'est le De libero arbitrio diatribè, tout en se plaignant à Warham: "Je me serais volontier abstenu de descendre dans l'arène des luthériens."27

Mais, de fait, il y est descendu à moitié et y a provoqué de malencontreux effets: l'insatisfaction des catholiques rigides, la colère grossière de Luther et la suspicion accrue des zwingliens de Bâle. Ainsi, en 1527, la situation d'Érasme est plutôt précaire, quoique sa réputation littéraire n'ait pas souffert. Toujours pragmatique, il comprend cependant qu'il risque de perdre la pension (irrégulière) de Charles Quint, les écus d'or que lui verse Ferdinand d'Autriche et les quelques revenus de la trésorerie de Tours dont il reconnaît avoir le plus urgent besoin.

Mais, d'autre part, il a toujours gardé l'ambition d'être plus que le pensionné des princes: il estime que la science, la fréquentation des modèles antiques et l'urbanité assignent à l'humaniste un rôle de conseiller auprès des grands. Conseiller, et non pas client, car la république des lettrés transcende, de droit sinon de fait, celle des citoyens. C'est pourquoi il a publié, à l'usage de Charles, en 1515, une sorte de speculum regis d'inspiration 
médiévale, décrivant le type parfait du prince chrétien, mu par la vertu; mais cette Institutio principis christiani a connu moins de succès que son manuel de savoir-vivre à l'usage des bourgeois: en effet, ce texte moralisateur ne propose aucune prise théorique sur les désordres inédits qui surgissent dans l'économie (effondrement des prix, passage à l'échange monétaire) ou dans le tissu social (paupérisation de masse, guerres confessionnelles, rébellions paysannes) qui assaillent son prince devenu l'empereur Charles Quint. Ce dernier déçoit Érasme qui le juge (aussi bien que François $\mathrm{I}^{\text {er }}$ et Henry VIII) trop jeune, trop ambitieux, trop brutal - bref, trop politique, dirions-nous aujourd'hui.

Dans cette perspective, la Pologne, plus lointaine et moins connue de lui, paraît offrir au conseiller bénévole un terrain d'application plus propice à son rêve. En bon humaniste, il se réjouit d'abord que ce royaume fasse partie du monde civilisé. Ce fut le premier rôle de ses contacts avec les lettrés polonais que de le rassurer à cet égard. Le Batave ignore cependant tout des facteurs internes ayant opéré pendant les six siècles de la genèse du "siècle d'or" en Sarmatie: il suppose que l'équilibre de paix qu'on y observe résulte d'influences occidentales, sans se demander pourquoi des causes semblables produisent ailleurs des effets aussi opposés. Quatre ans avant sa lettre à Sigismond, Érasme écrit à Decjusz:

Je félicite le peuple qui, décrié autrefois en raison de sa barbarie, est maintenant tellement florissant pour les lettres, les lois, les moeurs, la religion et tout ce qui soustrait à la honte de la barbarie, qu'il peut rivaliser avec les nations de premier rang et les plus renommées. $^{28}$

Et dès cette date, Sigismond lui apparaît comme une bien meilleure incarnation de l'idéal du prince chrétien que ne l'est devenu Charles Quint, perception sans aucun doute conforme aux données historiques:

... sur tous ces peuples, depuis la Vistule jusqu'à la Chersonèse de Tauride, de la mer Baltique aux Carpathes, le roi Sigismond exerce le pouvoir le plus large, lui qui non seulement l'emporte par toutes les qualités dignes d'un grand prince, mais encore s'est illustré par de nombreuses et grandes victoires remportées sur l'ennemi tartare et moscovite. Je ne sais vraiment pas si aucune autre fut plus indispensable à la protection de l'empire chrétien. ${ }^{29}$ 
L'admiration pour le caractère et pour l'oeuvre de Sigismond est certainement sincère. Néanmoins, Érasme a pris soin de vérifier que le roi s'est bien montré défavorable aux luthériens. Le 3 juin 1524, il fait savoir à Willibald Pirckheimer que "...à ce même moment, ${ }^{30}$ était chez moi le baron Hieroslaw, ${ }^{31}$ ambassadeur du roi de Pologne, qui m'a en très grande affection, mais ennemi irréductible de Luther, comme d'ailleurs le roi lui-même." ${ }^{32}$ Il se fait montrer par Hieronim Laski un exemplaire de l'édit royal du 24 juillet 1520 (réitéré en 1523), prohibant le commerce des oeuvres de Luther dans la république, mais il semble ignorer que le roi ne mit pas beaucoup de zèle à l'appliquer, non plus qu'à punir les familles qui continuèrent à envoyer leurs fils étudier dans les universités luthériennes, en contravention d'un autre édit royal. Comme on le voit, Érasme prend certaines précautions de peur d'être associé à une cause douteuse, mais sans se soucier de spécificité objective. Il n'a pas accès aux dossiers de la curie romaine, et il n'a pas lu Marsile de Padoue.

Et peut-être n'en prend-il pas suffisamment en ce qui concerne la dimension politique des affaires polonaises du jour et la qualité de ses informateurs. Il accord un crédit illimité aux lettrés (dont on a mentionné plus haut les liens avec la cour) et spécialement à Jan Laski, son invité et son ami, à propos duquel il écrit à Leonard Cox:

Jai pu connaitre et je connais mieux chaque jour le caractère de Jan Laski: il est tel que je me croirais assez heureux même avec ce seul ami. ${ }^{33}$

Né en 1499, Jan Laski a plutôt l'âge d'être le fils d’Érasme. ${ }^{34}$ En 1525 , lorsqu'il séjourne chez Érasme, il a déjà acquis une bonne expérience de l'Europe de l'ouest parce qu'il a étudié à Bologne, à Padoue et à Paris sans passer par l'université Jagellonne. Ordonné prêtre en 1521, il s'intéresse très tốt à tous les courants de la Réforme: ${ }^{35}$ il devait passer à la confession zwinglienne entre 1540 et $1542 .{ }^{36}$ Pour lors, il profite de son séjour chez Érasme pour fréquenter l'université de Bâle et, en autres, les cours de l'hébraiste Conrad Pellican, sympathique à la doctrine d'Oecolampade sur la Cène, cette même doctrine qu'Érasme a condamnée. C'est sans doute Laski qui rapporte à Érasme que Pellican se serait dit persuadé qu'au fond de son coeur, Érasme partageait la théologie eucharistique zwinglienne, sans vouloir l'admettre publiquement. Si Laski a effectivement joué ce rôle d'intrigant, il est largement responsable et de la rupture d'Érasme avec Pellican, et du départ forcé du maître pour Fribourg. Car, outré par ces 
rumeurs, Érasme rédige la "lettre à Pellican,"37 l’une des plus dure de sa correspondance, où, de nouveau contraint de descendre dans l'arène théologique, il se démarque de tous les hérétiques sur la question de la présence réelle dans l'eucharistie. On peut mesurer combien l'orthodoxie d'Érasme était alors peu évidente aux yeux des autorités de confession romaine puisque les érasmiens de l'ouest produisirent rapidement plusieurs éditions de cette lettre et s'assurèrent de sa diffusion, de manière à faire taire lessoupçons.

Or, Érasme s'en remet à Jan Laski pour toute la documentation sur le fondement de laquelle il compose la lettre à Sigismond. Il y a ici un premier malentendu sur les objectifs d'une telle intervention. D'un côté, Laski et la cour de Cracovie veulent un geste public, émanant d'une autorité morale non nationale, en vue de légitimer le traité de suzeraineté avec la Prusse luthérienne; il s'agit essentiellement de répliquer aux attaques du parti romain armé du droit canon, et de contrarier l'empereur que ce traité prive du fief de Prusse, sans parler de ses effets sur la sédition des princes luthériens: les Habsbourg seront donc heureusement occupés à réduire les désordres intérieurs. De son côté, Érasme est flatté qu'on invoque son nom et ses écrits à la lointaine académie Trzecieski. Il admire l'état de tolérance exceptionelle qui prévaut en Sarmartie. Il songe que François $1^{\text {er }}$ n'entend pas le latin et passe son temps à la chasse, tandis que l'humaniste Sigismond gère les affaires de près, calme les fureurs et encourage le dialogue. Volens, nolens, le nomade qui a toujours craint l'engagement se trouve donc de fait à prendre indirectement parti sur des questions de droit canon et de droit des gens. Voilà pour le contexte.

Quant au texte lui-même, il laisse une impression de malaise. La forme en est évidemment affable et flatteuse, mais aussi molle, vide et incertaine. Cette lettre a manifestement été sollicitée. ${ }^{38}$ Elle n'a pas d'objet précis. Elle se replie donc sur les figures rhétoriques, s'excuse de commencer et s'excuse de finir. C'est une lettre à un inconnu illustre, écrite sans nécessité apparente, à l'usage des tiers. Et quand on sait qu'Érasme s'est toujours montré un esprit incarné dans des humeurs et des images concrètes, un penseur qu'on pourrait dire "atmosphérique," c'est là un genre littéraire où il n'est pas à son meilleur.

Le contenu ${ }^{39}$ fait le panégyrique de la personne de Sigismond et de son oeuvre de roi. A soixante ans, celui-ci arrive alors exactement à la moitié d'un règne effectivement glorieux et brillant. On peut constater que toutes les idées "politiques" d'Érasme procèdent d'une éthique individuelle étendue à l'échelle des sujets collectifs que sont, à ses yeux, les nations, et qu'il ne 
peut penser autrement que sur le modèle psychologique et moral de la famille. Les événements évoqués sont alors tous réduits à une symbolique de l'ordre et de la paix domestiques. L'exposé, à prédominance narrative, est parsemé des motifs suivants: l'opposition entre civilité et barbarie (cette norme esthétique gouvernant toutes les autres); l'opposition entre "Empire chrétien" (au sens global médiéval) et mondes païens; l'opposition entre tranquillitas et guerre cruelle; enfin l'opposition entre dépravation cléricale et misssion évangélique de l'Église.

Les exemples anciens, païens et bibliques, traités sur le même pied, tiennent lieu à Érasme de principes. L'Europe étant à feu et à sang, il lui faut des sauveurs, tels Camille et Scipion l'Africain pour Rome, tels Moïse et Daniel pour les Hébreux. Érasme ne cherche pas la cause de l'excellence et du succès d'un chef. Hors de l'éthique, il a recours a la pensée magique:

Dieu sait toujours, dans les situations désespérées, faire surgir soudain comme par magie un homme doué d'une vertu singulière qui ramène à la tranquillité l'univers bouleversé.

Érasme dit avoir le présage que Dieu a ainsi désigné Sigismond pour maintenir la pax christiana, grâce à ses qualités "politiques": son grand âge qui lui vaut une précieuse expérience, sa sagesse, son autorité, sa prudence, sa magnanimité et enfin, son courage militaire.

De toute évidence, Érasme a un modèle comme le capétien Louis IX à l'esprit: un monarque patriarcal administrant un État de justice, loin de toutes les innovations de la modernité. Car il écrit que la plus importantes des vertus de Sigismond, c'est la piété, ainsi définie:

Le mot "piété" recouvre deux choses: l'amour pour la patrie, le zèle pour la religion; dans les deux domaines, tu t'es acquis les plus brillantes louanges. ${ }^{40}$

Aux yeux d'Érasme, les bonnes intentions font la bonne politique et un grand roi l'est d'abord parce que moralement bon. En attribuant à Sigismond le titre de Pater patriae, il réduit cette figure romaine du droit public à une dimension familiale intersubjective. Le seul élément moderne, dans sa typologie du roi chrétien, est ici la disparition du culte de la prouesse guerrière quasi gratuite qui tenait lieu de raison d'être au chef féodal et à ses barons. ${ }^{41}$ Ce furent d'abord les humanistes italiens de cour qui critiquèrent la valeur du courage-prouesse physique; Érasme la transforma en 
courage-force intérieure de type stolicien: le courage de faire et de maintenir la paix.

Ici, Érasme se situe aux antipodes de Machiavel. Il soutient que la paix politique s'obtient par la négation des passions que sont l'intérêt, l'appât du gain, l'ambition du pouvoir, la jalousie devant un plus puissant. Le concept érasmien d'utilitas reipublicae, dans cette lettre comme ailleurs, se trouve radicalement coupé du monde des rapports de forces, au lieu de définir les conflits et de chercher à les aménager. Nous pourrions dire que ce concept s'avère inefficace parce qu'apolitique.

Érasme reprend son fameux adage "Dulce quidem bellum inexpertis," en le limitant par la notion de necessarium bellum, c'est-à-dire la guerre qu'on ne saurait éviter sans tomber dans l'impiété. Sigismond, écrit-il, a eu raison de combattre militairement la barbarie des Scythes (Tatares) et des Turcs, non chrétiens: son royaume, grâce à ces victoires, forme le courageux rempart de l'empire chrétien civilisé. ${ }^{42}$ Mais on ne comprend pas pourquoi le roi aurait eu les mêmes mérites à vaincre la coalition des Valaques, des Prussiens et des Moscovites (Érasme omet ici de nommer le Saint-Empire parmi les coalisés). Cette guerre est-elle, de droit, également juste? Érasme vante seulement le roi d'avoir par ce moyen assuré la tranquillité du peuple polonais.

Il se contredit aussitôt en écrivant que Sigismond s'est surpassé à deux reprises en évitant de verser le sang chrétien, car un principe ancien et érasmien assimile la guerre entre chrétiens au scandale d'une guerre civile. La première occasion est précisément l'affaire prussienne où Érasme prête à Sigismond la seule motivation de l'amour de la paix:

... tu as préféré laisser au duc de Prusse une importante partie de ses états plutôt que de les soumettre en entier à ta domination, par la force, ce que tu aurais pu faire très facilement.

Pour faire bonne mesure et aussi, pour atténuer cet affront cinglant à l'autorité de Rome et de l'Empire, Érasme a d'abord rapporté le cas de la Prusse royale (intégrée au territoire du royaume) "que l'hérésie commençait à contaminer, [qui] est passée sous ta domination et a été corrigée." Historiquement, il est vrai que Sigismond avait le choix d'occuper et d'intégrer aussi bien la Prusse ducale que la Prusse royale et qu'il a choisi de ne pas procéder ainsi, malgré la détermination du parti anti-Habsbourg de sa cour dirigé par le vieux primat Jan Laski, l'irréductible. Cela eut 
constitué une politique plus prévoyante, à long terme; on sait, en effet que le duché revint à la branche aînée des Hohenzollern lors de la première crise dynastique, en 1618, puis qu'il s'érigea, en 1701, en un royaume dont la puissance militaire fut fatale à la Pologne, lors des partages. L'évangélisme d'Érasme ne permet de déduire ni une stratégie territoriale, ni une tactique diplomatique capables d'assurer une paix de fait. Quant au portrait de Sigismond en correcteur d'hérésie, c'est une image plutôt comique. L'expédition de Gdansk qu'évoque Érasme avait pour but de réduire une rébellion municipale et des actes réciproques de vandalisme dans les églises des deux confessions. Après avoir différé et négocié, Sigismond avait envoyé un détachement pour rétablir l'ordre civil et permettre aux tribunaux de juger les crimes prévus par le code, ce qui fut fait d'une manière exemplaire, c'est-à-dire durement, en prenant garde de ne réprimer aucune des deux confessions. Car Sigismond distingue clairement entre désordre religieux et désordre civil. ${ }^{43}$ Érasme, au contraire, ne reconnaît pas la distinction entre l'Église et l'État. Le malentendu, néanmoins, ne desservait pas les intérêts de la cour auprès des grandes puissances de l'ouest. Ce que Sigismond veut absolument éviter, c'est une conjonction de Rome et du Saint-Empire contre l'autonomie polonaise. Plus ou moins consciemment, Érasme a donc fourni le service qu'on attendait de lui.

L'autre occasion est encore plus délicate du point de vue diplomatique, et Érasme est encore moins au fait de ses nuances. Elle concerne la succession de Hongrie, ouverte par l'extinction des Angevins. Rappelons que Hongrois et Polonais sont des alliés traditionnels et perpétuels: on n'a jamais connu un seul cas de guerre entre ces deux couronnes qui ont parfois été portées conjointement par le même roi. La position polonaise n'a jamais varié: elle consiste à empêcher les Habsbourg d'occuper les trônes voisins, aussi bien en Bohême qu'en Hongrie d'ailleurs.

Or, en novembre 1526, Jan Zapolyai s'était fait élire roi de Hongrie par la petite noblesse, tandis qu'en décembre de la même année, la haute noblesse avait élu Ferdinand d'Autriche à la même couronne, convoitée par sa famille depuis le XIV ${ }^{e}$ siècle. Affrontement direct, donc, où les intérêts politiques de Sigismond et les intérêts personnels (matériels) d'Érasme ne peuvent, non plus, coïncider. Ferdinand déclare Zapolyai usurpateur. Ce dernier ose prêter hommage à Soliman II pour mieux résister au Habsbourg. Il pouvait certainement compter sur l'amitié personnelle de Sigismond qui avait épousé (en premières noces) sa soeur Barbara et allait lui donner en mariage sa fille Isabelle (née d'une seconde union avec Bona Sforza). De plus, comme 


\section{4 / Renaissance and Reformation}

Zapolyai, Sigismond s'était allié à la petite noblesse contre l'arrogance des magnats dans son propre royaume. Avec d'autres acteurs, une telle situation eut entraîné une guerre civile et internationale. Ce ne fut pas le cas. Les Hongrois, inquiets, offrent la couronne à Sigismond qui refuse cette pomme de discorde et délègue le chancelier Szydlowiecki pour régler l'affaire. $\mathbf{L a}$ médiation réussit: un traité partage alors le territoire entre les deux concurrents et les Hongrois eurent deux rois.

Érasme s'émerveille de l'héroïsme désintéressé de Sigismond qui a effectivement placé la paix au-dessus de l'ambition. Mais il commet l'erreur de désigner Zapolyai comme "Ungariae Rex," ce que son bienfaiteur Ferdinand n'apprécia guère. ${ }^{44}$ Encore ici, Érasme s'est manifestement fait manipuler par Jan Laski (et par la cour de Cracovie que celui-ci représente) dans une matière dont les tenants et aboutissants politiques lui échappent.

Le reste de la lettre a un caractère anodin et convenu. Évidemment, Érasme ne peut résister au plaisir d'y placer un passage violent et obsessif concernant les prévarications querelleuses des clercs, mais cette attaque parait gratuite $\mathrm{du}$ fait que l'auteur ne lui donne aucun contexte.

Pourtant, il faut encore relever l'énoncé de deux thèses, l'une politique et moderne, l'autre sociale et archaïque. La première soutient que pour garantir 'la paix entre les nations, chaque royaume devrait correspondre à un territoire d'un seul tenant. Les possessions lointaines, écrit Érasme, engendrent des guerres cruelles, toujours recommencées, comme le montrent l'exemple du Milanais pour la couronne de France, celui de l'Insubrie, etc. ${ }^{45} \mathrm{La}$ seconde thèse réitère, sans le fonder, le devoir de respecter l'ordre hiérarchique de toute société: chacun doit garder sa place, sans défier la structure du pouvoir, dit Érasme. Il faut comprendre, à défaut d'argumentation de l'auteur, que l'ordre établi est juste parce qu'établi depuis longtemps. Comme on connaît l'aversion d'Érasme pour les institutions et leur juridisme, il est facile de reconnaître ici l'une de ses principales objections contre la création d'une Église luthérienne. Et pourtant, le prince des lettres, roturier et bâtard, écrit cela même au prince héréditaire de Sarmatie, non sans jouir de l'autorité toute nouvelle que l'intellectuel moderne s'octroie à lui-même.

En somme, cette lettre à Sigismond, si elle ne contient rien de faux (hormis la correction d'hérésie) ignore tout des principes de tolérance religieuse et civile dont s'inspire le roi d'un État multi-ethnique et multi-confessionel. Elle ne tient pas compte des limites constitutionnelles du pouvoir royal dans la Pologne du $\mathrm{XVI}^{\mathrm{e}}$ siècle et attribue à la bonne volonté de Sigismond une modération inscrite dans le régime de la république nobiliaire et dans le 
contrôle qu'exercent les diètes. Le tableau idéal peint par Érasme repose donc sur une série d'équivoques.

La cour de Cracovie se déclara enchantée par cette lettre. A l'insu d'Érasme, Stanislaw Hozjusz, celui-là même qui accusa plus tard la Pologne d'être devenu "l'asile des hérétiques" et y prit la tête de la contre-Réforme, ${ }^{46}$ la fit publier aussitôt, ${ }^{47}$ sans en supprimer les indélicatesses à l'égard des Habsbourg. Cet opuscule, dédié à l'humaniste Tomicki, évêque de Cracovie et vice-chancelier du royaume contenait, en outre, la lettre à Pellican (dont on a parlé plus haut) ainsi que divers éloges d'Érasme signés par Logus, Werner, Lang, Cox et Hozjusz lui-même, le tout accompagné par un portrait du maître gravé sur bois. Il est intéressant de constater que la lettre à Pellican apparaissait encore nécessaire, en 1527, pour dédouaner l'orthodoxie romaine d'Érasme en Pologne et ailleurs. Mais quelle ironie dans ce couplage des deux lettres! C'est une lettre "théologique" qui est choisie alors qu'il réclamait à Laski qu’on diffusât ses oeuvres morales.

Le second événement marquant fut l'achat à Érasme de sa bibliothèque par la famille Laski qui lui en laissait l'usufruit sa vie durant. L'acte fut négocié et signé par Jan Laski le Réformateur pendant ce même fructueux séjour de 1525. A la mort d'Érasme en 1536, Andrzej Frycz Modrzewski (Modrevius) fut chargé d'en prendre possession et d'assurer son transport vers Cracovie.

On le voit, Érasme était sans doute mieux au fait de la situation politique et intellectuelle polonaise du temps que ne le sont la plupart des seizièmistes d'aujourd'hui, bien qu'il fut incapable d'en donner une analyse. Ses relations avec les cercles cracoviens n'étaient cependant pas tout à fait désintéressées. On sait ${ }^{48}$ avec quelle minutie frustrée il tenait le compte des dons, pensions, bénéfices et, plus souvent, de l'indifférence que lui avaient rapportés ses dédicaces aux grands, comme en témoigne le "Catalogue" de 1523. La famille Laski se montrait généreuse: les dédicaces à Hieronim Laski du De modo orandi Deum, en 1524, et de l'Institutio christiani matrimonii, en 1526, furent largement récompensées. Parfois, Érasme fut payé d'avance: après que le chancelier Szydlowiecki lui eut fait un don proportionné à sa haute situation, Érasme lui dédia le De lingua. Et il en avait été de même, en 1523, pour la dédicace de la Precatio dominica in Septem portiones distributa à Decjusz ${ }^{49}$ qui avait été jusqu’à commander cet écrit.

Entre 1520 et 1530, François $1^{\text {er }}$ tergiversait devant le projet de créer à Paris un collège trilingue où Érasme aurait pu se fixer pour prendre ses distances avec les soupçons qui pesaient sur ses travaux. S'étant entremis, le mal 
heureux Guillaume Budé fut contraint à des excuses épistolaires sans fin. En Pologne, au contraire, la cour, la chancellerie primatiale, les lettrés indépendants et ceux de l'université Jagellonne étaient unanimes à demander la venue d'Érasme. Sigismond souhaitait publiquement son installation en Pologne. Krzycki d'abord, en 1525, puis Jan Antonin, en 1526, deux correspondants fidèles, lui transmettent l'invitation. Érasme refusa, comme il refusa une offre semblable des Fugger pour Augsbourg. Il semble que, hors du couloir Rotterdam-Rome, il n'ait jamais cru pouvoir trouver l'art de vivre dans l'éclat des "bonae litterae" et des riches bibliothèques. Il ne visita jamais cette lointaine Pologne où sa renommée venait du fait qu'il incarnait des idéaux de modération, de dialogue et de tolérance que la culture polonaise avait élaborés sous la forme des sciences juridiques depuis le début du quinzième siècle. Le maître ignora toujours le fondement objectif de cette convergence.

On aurait cependant tort de croire qu'avec Érasme, la Pologne découvrait sa première version de la pensée érudite, antiquisante et critique. Pour situer le degré et les formes de cette nouveauté, il faut retracer les cadres de la production intellectuelle de la période antérieure et les mesurer à ceux de l'ouest européen.

Dès le XIV ${ }^{e}$ siècle, Casimir III le Grand ${ }^{50}$ avait obtenu d'Urbain V la bulle de 1364 instituant l'Université de Cracovie sur le modèle de Padoue et de Bologne. Casimir s'intéressait surtout à former les juristes dont avait besoin sa politique d'unification du royaume. Cette visée rencontrait les intérêts des savants: jusqu'aux partages de la Pologne, ses traditions juridiques originales restèrent la ligne directrice de la création intellectuelle et de l'apport polonais aux recherches occidentales. Une seconde bulle de Boniface IX, en 1397, ajusta l'université Jagellonne sur le modèle parisien par l'addition de la théologie. Professeurs et étudiants circulèrent largement suivant la filière du nord ou celle du sud, redoublant les circuits commerciaux du drap, des minerais, du blé, du bois, de la fourrure et de l'ambre.

Aux conciles de Constance et de Bâle qui furent des foires aux manuscrits et des tournois d'éloquence cicéronienne, les docteurs polonais se retrouvèrent face à plusieurs de leurs anciens maîtres. C'est ainsi qu'en 1414, Pawel Wlodkowic (Vladimiri) plaida contre l'Ordre Teutonique ${ }^{51}$ devant le padouan Zarabella qui lui avait enseigné le droit.

A partir de 1450 et jusqu'à la fin du $X V^{e}$ siècle, la filière italienne dominante amena dans tous les coins du royaume de Pologne une série continue de "mal sentans de la foi," éminents humanistes pour la plupart, 
qui trouvèrent là un refuge provisoire ou définitif: Filipo Buonaccorsi dit Callimaque, Stancaro, Biandrata, Ochino, Gentilis, Lelio puis Fausto Sozzini, etc. Il faut également tenir compte du fait qu'une autre filière de lettrés hellénistes donnait, depuis la défaite de Varna, en 1444, un accès direct à la Pologne aux réfugiés orthodoxes de Constantinople.

En ce milieu du $X^{e}$ siècle, la Pologne est donc loin d'apparaître comme un désert médiéval périphérique. Plus précisément, les variantes scolastiques dont Jan Dlugosz (Longinus) fut le dernier représentant, chevauchaient les courants néo-latins philologiques, philosophiques, poétiques et juridiques, comme c'était le cas en France ou en Angleterre. Le choix polonais de la romanité religieuse latine entretenait la participation à l'activité intellectuelle de l'ouest, y compris la dimension de critique des abus de l'Église dans un climat nettement réformateur. Par une exception remarquable, l'Université se montrait moins réfractaire qu'ailleurs aux méthodes et aux objets d'étude nouveaux: malgré des résistances issues de la faculté de théologie (aux cadres nécessairement plus âgés), on vit, dans le premier quart du $\mathrm{XVI}^{\mathrm{e}}$ siècle, les études bibliques, patristiques, grecques et judaïques s'intégrer en partie à la faculté des arts sans se placer en concurrence avec l'autorité universitaire.

Bien avant 1500, la figure humaniste du lettré, du juriste ou du poète indépendant avait acquis un statut, sinon une légitimité qui tendait à laïciser les formes du discours savant. Ces individus étaient recrutés à titre de secrétaires par les évêques et les magnats sénatoriaux, puis faisaient une carrière diplomatique dans les chancelleries primatiale et royale. Hieronim Laski et Andrzej Frycz Modrzewski illustrent la seconde génération de ce changement social.

L'engouement pour Érasme avait donc été précedé par l'italianisme impertinent dont s'amusait la cour. Callimaque ${ }^{52}$ avait organisé la sodalitas litteraria vistulana avec Conrad Celtis en 1498. Cet érudit florentin, épicurien, poète et partisan de la monarchie absolue, en quelque sorte machiavellien avant la lettre, ${ }^{53}$ vécut en Pologne de 1470 à sa mort, en 1496. C'est en 1472 que, malgré ses démêlés avec la curie romaine, ${ }^{54}$ Casimir IV en fit le précepteur de ses quatre fils, conjointement avec Jan Dlugosz. Le tandem était judicieux: il plaçait les princes au carrefour de l'humanisme italien et des traditions polonaises séculaires. L'aîné de ces princes régna sur la Bohême et sur la Hongrie, ${ }^{55}$ les deux suivants occupèrent brièvement le trône de Pologne; ${ }^{56}$ Sigismond I le Vieux fut le dernier des fils de Casimir à monter sur le trône polonais, d'où son surnom. 
Callimaque avait trouvé à Cracovie un milieu déjà instruit des nouveautés culturelles par les voyages et par des études en Italie; parmi ces lettrés, on peut identifier Grzegorz Sanok (z Sanoka) Mikolaj Hussów (Hussowski), Maciej Drzewicki, Zbigniew Olesnicki et Jan Ostroróg. Les premières presses ouvrirent l'ère de l'imprimerie à Cracovie en 1474, soit deux ans après celles de Paris.

Quand la filière du nord suscita la mode érasmienne, les intellectuels polonais perçurent donc nettement la rupture avec l'italianisme. Il sentirent qu'Érasme refusait le formalisme et le maniérisme de cour, qu'il rompait avec le "paganisme" padouan et voulait soumettre les sciences du texte à la promotion de l'idéal évangélique, enfin qu'il renouvelait l'usage du néo-latin en lui donnant une forme libre et ouverte.

Les deux courants continuèrent néanmoins d'alimenter la pensée et les arts polonais. Sigismond le Vieux était familier de l'humanisme italien grâce à son précepteur, le pragmatique Callimaque, mais surtout à cause des années de jeunesse passées chez son frère à la cour de Hongrie où prévalaient la musique, l'architecture et les arts décoratifs italiens. Devenu roi, en 1506, il entreprit de grands travaux d'architecture religieuse et civile, dont l'extension et la rénovation du château royal de Wawel, mobilisant des artistes et artisans d'Italie. Et lorsqu'en 1518, il se remaria à Bona Sforza, duchesse de Bari, Cracovie avait déjà un visage italien. Ce n'est donc pas ce mariage qui a italianisé la cour polonaise mais c'est plutôt l'italianisme de la cour qui a favorisé ce mariage. Le même Sigismond demande l'appui politique d'Érasme et l'invite à s'installer en Pologne: il manifestait par là un virage où la reine ne le suivit pas.

Il nous apparaît qu'Érasme n'a jamais pris l'exacte mesure de cette conjoncture historique sur laquelle un séjour à Cracovie l'eût renseigné. C'est pourtant pour la cause polonaise, on l'a vu, qu'il prit l'engagement politique le plus concret de sa carrière.

\section{Université d'Ottawa}

\section{Notes}

Note: Je voudrais exprimer mes remerciements à mon collègue, le professeur James A. Parente, de l'Université de Chicago, ainsi qu'au professeur Juliusz Domanski, de l'Université de Varsovie, dont les commentaires óclairés m'ont servi dans la révision de ce texte.

1 Soit entre la publication par Érasme des Annotationes de Valla et la condamnation d'Érasme par IIndex, sous Pie IV.

2 Johan Huizinga, Erasmus and the Age of Reformation (1924), trans. F. Hopman (Princeton NJ.: Princeton University Press, 1984), p.106. 
3 Cf. Allen, P.S., Allen H.M., Garrod, H.W, Opus epistolarum Des. Erasmi Roterodami (Oxford: Oxford University Press, 1906-1967), tome V, Ep. 1488.

4 Maria Cytowska ed., trad, int. et notes, Korespondencja Enazma z Rotterdamu z Polakami, P.I.W. (Warszawa, 1965), p.8.

5 Cf. "The Executionist Movement in Sixteenth century Poland: a Cultural Mirror" in Proceedings of the Unitarian Universalist Historical Society, (Society for Reformation Research XX-II, 1985-6), pp. 33 à 45.

6 Cf. Janusz Pelc, Europejskasc $i$ Polskasc literatury naszego renesansu, (Czytelnik: Warszawa, 1984),pp.132-133.

7 Ce surnom qu'il acquit après 1540 a ici l'utilité de le distinguer de son oncle du même prénom.

8 Connu d'Érasme qui s'en informe sans lui \&́crire directement, par ex. voir $E p .1821$.

9 Cf. Ambroise Jobert, De Luther d Mohila. La Pologne dans la crise de la chrétienté 1517-1648 (Institut d'études slaves: Paris, 1974), pp. 48 sqq.

10 D'où ils attaquaient aussi la Lituanie ce qui explique l'union des deux couronnes de 1413.

11 Selon la tradition juridique polonaise, le duc Conrad de Mazovie leur avait accordé un droit de séjour limité et révocable.

12 Au Concile de Constance, Pawel Wlodkowic (Vladimiri) soutient que le document invoqué par l'Ordre est un faux.

13 La Pologne n'hésita pas å utiliser des mercenaires hussites très efficaces sur le front du nord, ce qui lui fut reproché au débat de Constance.

14 Cf. Oskar Halecki, Histoire de Pologne, éd. Roy (New York et Montréal, 1945), pp. 140 à 147.

$15 \mathrm{Cf}$. Tadeusz Wyrwa, La pensée politique polonaise d l'époque de l'Humanisme et de la Renaissance, Librarie polonaise (Poets and Painters Press: Paris/Londres, 1978), pp. 353 à 362; Zygmunt Wojciechowski, "Le traité de Cracovie de 1525," in Revue historique, 207 (1952), pp. 15 sqq.

16 La liberté de culte qui avait toujours prévalu lui fut officiellement octroyée en 1557-1558.

17 A quoi Sigismond répondit: "Laissez moi être le pasteur des boucs et des brebis" pour marquer la distinction entre l'Église et l'État. Cf. Jobert, op. cit, p.48.

18 Cf. Ep. 1820 au chancelier Szydlowiecki, du 16 mai 1527: "Jai aussi écrit quelques mots à ce sujet au glorieux roi de Pologne, Sigismond, comme les lettres de ce brillant jeune homme, Jean Laski, m'y avaient invité plus d'une fois. Il l'a sollicité avec tant de passion que je crois que cette ardeur doit avoir quelque bonne raison." In La correspondance d'Érasme, sous la dir. de Alois Gerlo, Institut universitaire pour l'étude de la Renaissance et de l'humanisme, Presses de l'Université libre de Bruxelles, tome VII, 1978, p. 84. Ces lettres de Jan Laski sont perdues. Mais on peut constater qu'Érasme reconnait, après la rédaction de sa lettre, ne pas avoir saisi le rôle politique exact qu'on lui a fait jouer.

19 Pour cette première des deux lettres d'Érasme à Sigismond, nous avons utilisé le texte latin de l'édition Allen, Ep. 1819, op. cit, tome VII, pp. 59 à 65 et nous citons le texte français de La Correspondance d'Érasme, op. cit., tome VII, pp. 77 à 84 . Sur cette question, cf. Claude Backvis, "La fortune d'Érasme en Pologne" in Colloquium Erasmianum (Mons, 1968) et Jean-Claude Margolin qui a donné une traduction et un commentaire de cette lettre in Guerre et paix dans la pensée d'Érasme (Paris, 1973), pp. 294 à 305; voir aussi George H. Williams, "Erasmianism in Poland. An account and an Interpretation of a Major, Though ever Diminishing, Current in Sixteenth-Century Polish Humanism and Religion, 1518-1605”, in The Polish Review 22 (1977), pp. 4 à 50.

20 Allen, O.E. IV, $E$ p. 1232, p. 575. C'est l'image de Jules II qui inspire les envolées d'Érasme.

21 Allen, O.E. VII, Ep. 1488; C.E. VII, p. 674.

22 Les autorités municipales bâloises l'avaient formellement consulté sur cette question.

${ }^{23}$ Allen, O.E. VII, Ep. 1821; C.E. VII, p. 87. 


\section{0 / Renaissance and Reformation}

24 Delumeau et Lecler s'entendent pour condamner le simplisme théorique d'Érasme, aveugle au besoin de précision doctrinale qui sous-tend l'esprit reformateur catholique aussi bien que protestant; cf. Joseph Lecler, Histoire de la tolérance au siécle de la Réforme (Aubier-Montaigne: Paris, 1955), tome I, pp. 144-145, et Jean Delumeau, La civilisation de la Renaissance (Arthaud: Paris, 1984), p. 130.

25 Richard Popkin l'attribue à un semi-scepticisme de principe; cf. The History of Scepticism from Erasmus to Descartes (Assen: Van Gorcum, 1960), pp. 4 à 7.

26 Allen, O.E. VII, Ep. 1451; C.E. VII, p. 587.

27 Allen, O.E. VII, Ep. 1488; C.E. VII, p. 675.

28 Allen, O.E. V, Ep. 1393; C.E. V, p. 437.

29 Ibidem. A noter l'emploi de l'expression "empire chrétien" dans son acception médiévale selon laquelle le monde civilisé ne reconnaît que deux autorités: l'Église et l'Empire.

30 Où Luther lui ecrivait.

31 Hienonim Laski.

32 Allen, O.E. V, Ep. 1452; C.E. V, p. 591.

33 Allen, O.E. VII, Ep. 1824; C.E. VII, p. 91.

34 Sur la carrière de Jan Laski le Réformateur, cf. Ambroise Jobert, op. cit., pp. 95 à 120 et Jean-Claude Margolin; "Erazmianizm Jana Laskiego" in O.i R. IX, 1964, pp. 75 à 84.

35 Il entretiendra des relations suivies avec Melanchton et avec Calvin.

${ }^{36}$ La contre-Réforme y verra plus tard une preuve de la néfaste influence d'Érasme sur Laski, comme sur d'autres réformés polonais.

37 Allen, O.E. VI, Ep. 1637 du 15 mai 1525.

38 Cf. Allen, O.E. VII, Ep. 1821 du 17 mai 1527 à Jan Laski: "Jai ócrit au Roi comme tu me l'as tant de fois demandé, mais tu trouveras ci-jointe une copie conforme: tu pourras donc faire disparaitre la lettre si tu le juges nécessaire; c'est qu'il est difficile d'écrire aux rois!" Cf. C.E. VII, p. 86. II est en effet très difficile d'écrire aux rois lorqu'on est dépourvu de théorie politique. A noter que l'intermédiaire a pu recevoir cette copie non datée ce qui expliquerait pourquoi la réponse de Sigismond place la date de la lettre d'Érasme au mois de juin.

39 La lettre 1819 compte 214 lignes latines dans l'edition Allen (VII, pp. 59 à 65) et 260 lignes françaises dans la traduction Gerlo (VII, pp. 77 à 85).

40 "Pietatis nomen duo complectitur, amorem patriae et studium religionis. In utroque genere summa tua laus eluxit..."

41 Cette représentation survivra longtemps: Henry II de Valois meurt dans un tournoi contre Montmorency, en 1559 et, au XVII ${ }^{e}$ siècle, Richelieu doit interdire les duels où se décime la jeunesse noble.

42 Sur ce mythe qui occulte les puissantes communautés orthodoxes (russes et grecques), arméniennes, juives, musulmanes païennes, etc., cf. Norman Davies, God's Playground. A History of Poland (Oxford: Clarendon Press, 1982), tome I, ch. VI "Antemurale: The Bulwark of Christendom."

43 Ce sont les souverains Sigismond et Bona qui fondent, en Prusse ducale, à même le trésor de la maison royale de Pologne, 1'Université luthérienne de Królewiec (Konnigsberg) en 1544, et qui placent Kulwiec au rectorat. Il ne fait aucun doute que le roi ne craint pas la coexistence des deux confessions. L'histoire polonaise lui a appris depuis plusieurs siècles que l'absence d'unité religieuse n'entraine pas l'effrondrement de l'État.

44 Éditant lui-méme sa lettre à Sigismond, en 1529, Érasme corrigea l'expression qui devint: "qui a usurpé le titre de roi."

45 Il ne mentionne pas le pouvoir espagnol sur les Pays-Bas.

46 Sans que jamais se démente sa loyauté au souvenir d'Érasme.

47 Chez H. Wietor, Cracovie, 1527, sous le titre de Des. Erasmi Roterodami epistola ad inclytum Sigismundum regem Poloniae etc. mire elegans, in qua horum temporum conditionem graphice 
describit. Ejusdem ad amicum quendam Epistola, pia juxta et elegans, qua calumniam sibi intentatam, quod aliter atque par erat de sacratissimo Christi corpore sentiret, scite breviter refellit. Allen dit en avoir consulté un rare exemplaire à la bibliotheqque Zamoyski, à Kómik, le 8 juillet 1922; cf. Allen, O.E. VII, pp. 59-60. Je tiens à remercier Madame Jolanta Lenkiewicz, conservateur, qui m'a facilité l'accès à l'excellent exemplaire du fonds Czartoryski de Cracovie.

C.. Jobert, op. cit., p. 54.

50 Demier roi de la dynastie Piast 1333-1370, allié aux Angevins de Hongrie qui le rapprochèrent du réseau italien.

51

Cf. Norman Davies, op. cit, pp. 165-166.

52

Cf. Wyrwa, op. cit, pp. 203 \& 224.

53

Cf. Les Consilia Callimachi qui lui sont attribués.

54 Paul II exigeait son extradition que le roi refusa.

55 Ladislas de Bohême (1471) et de Hongrie (1490-1516).

56

Jan Olbracht (1492-1501) et Aleksander $\mathrm{fr}^{\mathrm{r}}$ (1501-1506). Les thèses absolutistes de Callimaque se firent sentir sous Jan Olbracht, le seul monarque de l'histoire nationale qui ait tenté de les imposer, et $c e$, sans succès. Ostroróg l'avait appuyé dans cette direction et garda le désir d'un pouvoir royal fort. 